\title{
Preferências dos adolescentes sobre os cuidados de saúde
}

\author{
Adolescents' preferences regarding health care
}

Maria da Graça Vinagre (http://orcid.org/0000-0002-9610-9806) ${ }^{1}$

Luísa Barros (https://orcid.org/0000-0002-5075-0104) ${ }^{2}$

${ }^{1}$ Escola Superior de Enfermagem de Lisboa. Av. Prof. Egas Moniz s/n. 1600-190 Lisboa Portugal. gvinagre@esel.pt

${ }^{2}$ Faculdade de Psicologia,

Universidade de Lisboa.

Lisboa Portugal.

\begin{abstract}
Adolescents underutilize health services, especially for monitoring purposes, which represents a concern for professionals. Since adolescence is a crucial phase in acquiring healthy behaviors and attitudes that facilitate access to these services, finding ways to respond to the specific needs of adolescents through their participation is an important investment in the well-being of future generations. This is an exploratory, descriptive and qualitative study with two phases, seeking to identify and analyze adolescentser \#39; ideas and preferences about healthcare. Eight group interviews were conducted with 64 adolescents aged 13 to 18 years: four focus groups (phase 1) and four nominal groups (phase 2). The data was submitted to content analysis. The adolescents revealed both favorable and unfavorable opinions, highlighting a set of preferences regarding service conditions, such as short waiting periods, more comfortable and less crowded settings. Concerning the professionals' attitudes, they emphasized their technical competencies, such as knowledge and experience, although combined with interpersonal skills. These findings support the need for changes in organizational practices, and particularly in the attitudes of the professionals.

Key words Adolescents, Healthcare, Healthcare services, Qualitative research
\end{abstract}

Resumo Os adolescentes subutilizam os serviços de saúde sobretudo para efeitos de vigilância, o que constitui uma preocupação para os profissionais. Porque a adolescência é uma fase crucial à aquisição de comportamentos saudáveis e de atitudes facilitadoras do acesso a estes serviços, por isso encontrar formas de responder às necessidades especificas dos adolescentes, através da sua participação, constitui um importante investimento no bem-estar das gerações futuras. Este estudo exploratório e descritivo, de natureza qualitativa, com duas fases, teve como objetivos identificar e analisar as ideias e preferências dos adolescentes sobre os cuidados de saúde. Realizaram-se oito entrevistas de grupo com 64 adolescentes dos 13 aos 18 anos: quatro grupos focais (fase 1) e quatro grupos nominais (fase 2). Os dados foram tratados através da análise de conteúdo. Emergiram opiniões favoráveis e desfavoráveis. Nas suas preferências destacaram-se, nas condições dos serviços, haver menos tempo de espera e ambientes mais confortáveis e menos lotados; nas atitudes dos profissionais privilegiaram as competências técnicas, como o saber e a experiência, embora associadas às competências relacionais. Os resultados suportam a necessidade de mudanças nas práticas organizacionais e sobretudo nas atitudes dos profissionais. Palavras-chave Adolescentes, Cuidados de saúde, Serviços de saúde, Pesquisa qualitativa 


\section{Introdução}

Investir na saúde dos adolescentes poderá traduzir-se em importantes ganhos não só para esta etapa do ciclo de vida como também para as gerações seguintes ${ }^{1-3}$. Sabemos que a adolescência é um período de confronto com mudanças de ordem biológica, cognitiva, emocional e sociocomportamental e o facto de terem de fazer opções e tomar decisões que têm repercussões nos seus projetos de vida, gera necessidades específicas às quais os cuidados de saúde devem dar resposta ${ }^{4}$.

No entanto, assiste-se a uma escassa procura dos Cuidados de Saúde Primários pelos adolescentes $^{5}$, nomeadamente em Portugal ${ }^{6}$. A subutilização destes serviços assume maior importância pela notória prevalência de comportamentos de risco neste grupo etário, responsável por uma elevada morbilidade que advém das consequências negativas destes comportamentos e que se mantém como uma forte ameaça à saúde e bem -estar dos jovens ${ }^{2,7}$. A necessidade do adolescente aprender a lidar com os riscos comuns nesta etapa, associada às atuais instabilidades sociais, e o facto de ser uma idade crucial para a aquisição de comportamentos saudáveis e de padrões de utilização dos serviços que têm tendência a manterse na idade adulta ${ }^{8}$, têm incentivado o estudo do problema, podendo constituir um investimento importante ao nível da saúde pública9 .

Atualmente as políticas preconizam estratégias de intervenção mais focadas na promoção da saúde e mais responsivas às necessidades dos adolescentes $^{2,9}$, sugerindo a inclusão dos adolescentes como protagonistas nestes cenários que lhes dizem respeito ${ }^{2,10}$. No entanto a investigação, envolvendo os jovens no sentido de orientar tais mudanças, tem sido bastante escassa, particularmente no nosso país.

A literatura internacional tem identificado um conjunto de determinantes, onde se salientam as condições dos serviços de saúde e as atitudes e comportamentos dos profissionais como tendo um papel decisivo na utilização destes serviços pelos adolescentes ${ }^{3,11}$.

Os resultados de alguns estudos focam-se na importância de fatores relacionados com o saber e a competência dos profissionais de saúde, e em aspetos da comunicação e da relação com os adolescentes como a confiança, o respeito, a compreensão, a privacidade e a confidencialidade ${ }^{12,13}$, argumentando que quando a experiência direta com os profissionais é positiva, aumenta a satisfação e, por consequência, a probabilidade de os jovens voltarem a procurar os serviços de saúde, prevenindo consequências negativas ao nível do seu bem-estar ${ }^{9}$. Outros salientam a preocupação dos adolescentes com as condições de higiene, evocando o problema da transmissão das doenças $^{14}$, bem como a necessidade de os serviços serem de fácil acesso, atrativos e confortáveis, onde o ambiente estimule os adolescentes a falarem sobre os seus problemas e sentimentos, ou mesmo a abordarem determinados comportamentos que os podem expor ao risco ${ }^{15}$.

Este conhecimento tem motivado esforços no sentido de desenvolver e implementar serviços de saúde mais próximos e adequados ao desenvolvimento e às necessidades específicas dos jovens, tendo como referência os critérios definidos pela World Health Organization para os designados Adolescent Friendly Health Services ${ }^{16,17}$. Ou seja, serviços que cumpram critérios de qualidade, atendendo a um conjunto de características e condições: sejam acessíveis, aceitáveis, apropriados, efetivos e que respeitem a equidade ${ }^{17}$. Neste contexto, cuidados de saúde de qualidade correspondem a cuidados centrados na pessoa do adolescente, ou seja, que respeitam e respondem às preferências, necessidades e valores dos adolescentes ${ }^{18}$.

Todavia, e como reforçam alguns autores, ainda sabemos muito pouco sobre o que pensam e sentem os adolescentes sobre estas questões ${ }^{19,20}$, e as políticas e programas de saúde são ainda maioritariamente baseados nas ideias dos adultos. Os serviços são desenhados por adultos para adultos, onde são consideradas sobretudo as suas necessidades, e são os jovens que referem tendencialmente piores experiências quando comparados com outros grupos etários ${ }^{21}$.

No sentido de contribuir para cuidados de saúde mais adequados às necessidades e desejos dos adolescentes portugueses, e seguindo as atuais recomendações das políticas de saúde ${ }^{2,10,17}$, considera-se fundamental dar voz aos adolescentes, começando por saber o que nos têm a dizer sobre estas questões. Também alguns autores, como Hargreaves $^{19}$, aconselham os políticos e os profissionais de saúde a ouvirem e respeitarem as opiniões e sugestões dos jovens, ("learning to listen”), defendendo que tal é imprescindível para haver sucesso no objetivo de melhorar os cuidados de saúde dirigidos aos adolescentes ${ }^{19}$. O autor refere igualmente a necessidade de realizar estudos qualitativos para aumentar a compreensão da experiência dos jovens com os cuidados de saúde, e estudos quantitativos para aumentar a quantidade dos dados recolhidos ${ }^{19}$.

Reconhecendo a importância das ideias dos jovens e acreditando na sua mais-valia para a 
mudança das práticas de cuidados, com este estudo pretendeu-se identificar e analisar as ideias e as preferências dos adolescentes sobre os cuidados de saúde partindo das suas experiências com os serviços e com os profissionais.

\section{Métodos}

O presente estudo inclui duas fases e integra um projeto de investigação mais vasto. Trata-se de um estudo exploratório, descritivo e predominantemente qualitativo cujas fases são sequenciais: na primeira utilizaram-se entrevistas de Focus Groups ${ }^{20}$ e, na segunda, entrevistas de grupo com recurso à técnica do grupo nominal (Nominal Group Technique - NGT), com uma amostra de características semelhantes.

A Fase 1, com os grupos focais permitiu aceder às ideias, crenças, atitudes e algumas experiências em relação aos cuidados de saúde dos adolescentes $^{20}$. Para Morgan, o que distingue os grupos focais é a utilização explícita da interação grupal para produzir dados e aceder a perspectivas que dificilmente seria possível de outra forma $^{22}$. A interação que se gera na discussão favorece o envolvimento ativo dos participantes permitindo expressar, explicar e até defender as suas opiniões num contexto dinâmico, coletivo e num ambiente não ameaçador que estimula a espontaneidade ${ }^{22}$. Esta técnica de recolha de dados tem-se revelado de grande utilidade em estudos com adolescentes na área da saúde $\mathrm{e}^{13-14,20}$.

A necessidade de clarificar, sistematizar e priorizar as ideias que emergiram dos grupos focais motivou a realização de novas entrevistas grupais na Fase 2, mas agora num formato mais estruturado, recorrendo à Nominal Group Technique (NGT), no sentido de obter informação estruturada e ordenada por prioridades face às questões colocadas pelo investigador. Esta técnica favorece a participação de todos os elementos do grupo, minimizando a influência do moderador e tem sido também muito utilizada com adolescentes $^{23}$, por vezes em associação com os grupos focais $^{14}$. É um processo de tomada de decisão em grupo que permite a expressão das ideias dos diferentes elementos bem como a obtenção de consenso no grupo, de forma democrática, por via da estrutura e sequência de um conjunto etapas sequenciais $^{14,23}$. O objetivo foi o de obter informação estruturada, com clarificação de indicadores, sobre as características e atitudes preferidas nos profissionais de saúde pelos adolescentes.

\section{Instrumentos e Procedimentos}

Para a recolha de dados foram utilizados três instrumentos: um breve questionário individual de autopreenchimento para caracterização sociodemográfica das duas amostras e informações sobre a situação de saúde, e dois guiões diferentes para a condução das entrevistas de cada fase. Os tópicos abordados incluíram as experiências com os serviços de saúde, aspetos mais ou menos apreciados, e as características e condições desejadas nos profissionais e nos serviços. Por exemplo, na fase $1^{20}$, Como seria para vós um serviço de saúde ideal?, Para vós o que é um bom profissional de saúde? Na fase 2, Quais as características e atitudes que vocês acham mais importantes, ou preferem encontrar, num profissional de saúde?

O estudo obteve a aprovação dos Conselhos Executivos das escolas, tendo sido solicitada autorização com consentimento informado aos adolescentes e encarregados de educação. De acordo com o protocolo, as sessões foram todas gravadas em vídeo, com a autorização prévia dos participantes.

Nas entrevistas, de ambas as fases, cada grupo teve início com o acolhimento aos participantes, a apresentação da moderadora e assistente e dos objetivos do estudo. Foi realçada a importância da participação de todos, e a ideia de que não existiam respostas certas ou erradas, mas sim opiniões diferentes e todas muito importantes. Foram dados os necessários esclarecimentos quanto a regras e considerações éticas que estas metodologias envolvem. Depois destas informações, iniciava-se a sessão com uma atividade "quebra-gelo", com o objetivo de os envolver e facilitar a interação, o que resultou salientando-se um ambiente mais lúdico nos grupos dos adolescentes mais novos. Foi recordado que a colaboração era voluntária e que, apesar de ser muito útil, não era obrigatória, havendo sempre a possibilidade de saírem da sala sem justificar o motivo.

Especificamente, na realização dos grupos focais (Fase 1) foram respeitadas as etapas propostas por Morgan ${ }^{22}$ : o planeamento, recrutamento, moderação e análise dos dados. Os 33 adolescentes que participaram, nesta primeira fase, foram organizados em quatro grupos mistos, de rapazes e raparigas. Dois grupos com adolescentes de 13 e 15 anos, e outros dois entre os 16 e 18 anos, variando a composição entre sete a nove participantes por grupo. As entrevistas foram realizadas nas escolas e tiveram uma duração média de 70 minutos. 
Nos grupos nominais (Fase 2), para além do planeamento e recrutamento, seguiram-se as cinco etapas preconizadas pelos autores ${ }^{23}$ a seguir durante as entrevistas. O moderador, após o acolhimento já referido, expôs ao grupo as questões relacionadas com o tema em discussão e os adolescentes pensaram em silêncio nas respostas e registaram individualmente as suas ideias numa folha de registo preparada para o efeito. Posteriormente, cada um, por sua vez, apresentou ao grupo, uma das ideias que havia escrito e, uma a uma, percorrendo os diferentes elementos, foram apresentando todas as suas ideias, enquanto o moderador ia registando a informação no quadro negro visível a todos os participantes. Passou-se à discussão em grupo para clarificação das ideias obtidas, promovendo a participação de todos os elementos do grupo.

Após a clarificação, cada elemento, individualmente e em silêncio, selecionava cinco dos aspetos que considerava mais importantes, entre a totalidade da lista obtida em grupo, e atribuía uma pontuação a cada aspeto selecionado, ordenando-os pelo grau de importância que lhes conferia. Esse registo foi feito em folha de papel preparada para o efeito.

Por fim, partilhavam-se os resultados finais, mais uma vez registrados no quadro negro e após discussão, na maioria dos grupos, muito participada e disputada, encontrava-se o consenso em relação ao conjunto de ideias mais importantes para o grupo em questão. Os participantes assistiam à contagem da pontuação e tinham a oportunidade de confirmar e manifestar mais uma vez a sua concordância com o resultado final.

Os 31 adolescentes que participaram, nesta Fase 2, foram organizados igualmente em quatro grupos mistos, respeitando as mesmas idades da Fase 1, e a composição dos grupos variou entre seis e nove adolescentes. As entrevistas foram realizadas nas escolas com uma duração média de 90 minutos.

Em geral, e em ambas as fases, as discussões decorreram com entusiasmo. Os adolescentes revelaram muito interesse pelos temas propostos e os grupos foram bastante dinâmicos. Partilhando das ideias de Morgan ${ }^{22}$ que os incentivos motivam a comparência dos participantes nos grupos e estimulam a participação, particularmente neste grupo etário, no final de cada sessão foram distribuídos alguns brindes (sobretudo material escolar), anunciados previamente no convite para colaborarem no estudo.

Após terem sido realizados os quatro grupos em cada fase ( 1 e 2), por haver saturação na maioria dos dados recolhidos, passou-se à respetiva análise.

\section{Análise dos dados}

Os dados foram tratados recorrendo à análise de conteúdo temática categorial segundo Bardin $^{24}$. O corpus de análise resultou das transcrições integrais das entrevistas. Procedeu-se à codificação, organização e reorganização da informação encontrando o seu sentido, atribuindo significado ao seu conteúdo, e construindo uma grelha de análise com temas, categorias, subcategorias e unidades de registo ilustrativas.

Este processo de codificação foi realizado pela investigadora e por três juízes, procedendo-se à comparação das diferentes codificações, para assegurar a consistência e o rigor do mesmo. Na Fase 1, na contagem das frequências considerou-se o número de adolescentes que fizeram referência aos vários assuntos (extensividade) independentemente do número de vezes que foram referidos. As percentagens foram calculadas em função dos 33 adolescentes que participaram nos grupos focais.

Na Fase 2, seguiram-se os métodos de análise e de cotação preconizados para a $\mathrm{NGT}^{23}$. Um processo que envolveu a transcrição das gravações e respetiva validação através da informação escrita pelos adolescentes durante a realização dos grupos. De seguida procedeu-se à organização da listagem das características e atitudes dos profissionais consideradas mais importantes por cada grupo de adolescentes, com a respetiva cotação atribuída individualmente e validada em grupo para cada afirmação produzida. Para a atribuição de uma pontuação, pelos adolescentes, foi utilizada uma escala tipo likert que variava entre 1 e 5, do menos ao mais importante. No final, construíram-se quatro quadros, com a informação produzida e hierarquizada por cada grupo de adolescentes. Posteriormente agruparam-se os dados por grupo etário e por ordem decrescente de pontuação.

\section{Resultados}

Quanto à caracterização sociodemográfica dos 64 adolescentes que participaram no estudo (33 na Fase 1 e 31 na Fase 2), 35 eram raparigas e 29 rapazes, com idades compreendidas entre os 13 e 18 anos, que frequentavam o $8^{\circ}$ ano, o $11^{\circ} \mathrm{e}$ o $12^{\circ}$ ano de escolaridade de duas escolas públicas de Lisboa. Pertenciam na sua maioria ao ní- 


\section{Fase 1: Grupos Focais}

Na primeira fase, da análise de conteúdo produzida nos grupos focais, emergiram os temas os adolescentes e os recursos de saúde; e os adolescentes e os profissionais de saúde ${ }^{20}$. Apresenta-se o conjunto de categorias e subcategorias, construídas a partir das unidades de registo, para cada tema.

O primeiro tema, (Tabela 1), os adolescentes $e$ os recursos de saúde, inclui três categorias. Os adolescentes reportam-se ao tipo de serviços que procuram, bem como aos motivos porque o fazem e a quem recorrem. Pronunciam-se avaliando negativamente as condições de atendimento e funcionamento dos serviços de saúde e assinalam as suas preferências em relação aos mesmos ${ }^{20}$.

Os Hospitais e os Serviços de Atendimento Permanente (SAP) são os serviços a que mais recorrem para resolver situações de urgência, ace- der a consultas de especialidade e também para internamento, sendo a doença e os acidentes os principais motivos de procura. Os Centros de Saúde são procurados para a vacinação e consultas de vigilância, e também por doença. A consulta para adolescentes é assinalada como um espaço relevante que permite obter apoio sem a interferência dos pais. Curiosamente, as farmácias são identificadas como um recurso de saúde de fácil acesso e que responde bem aos pequenos problemas do quotidiano.

Todos os grupos identificaram os problemas psicológicos como um motivo relevante para procurar os serviços de saúde, explicitando as "depressões" e os "traumas” como uma razão importante, lembrando que a doença não é só os problemas físicos. O profissional que todos procuram é o médico, sendo o médico de família a referência mais frequente.

A avaliação do funcionamento geral dos serviços foi sobretudo negativa. Longos períodos de espera para marcação de consulta e atendimento, com más condições físicas e ambientais, como referiram vários adolescentes (A), as salas são desconfortáveis (A23); estava muito frio (A3); fazendo referência à sobrelotação e salientando o risco de contágio as urgências... aquilo fica muito cheio, muito calor, demora muito... uns que

Tabela 1. Tema I - Os adolescentes e os recursos de saúde. Lisboa; 2016.

\begin{tabular}{|c|c|c|c|c|}
\hline \multirow{3}{*}{\begin{tabular}{l}
\multicolumn{1}{c}{ Categorias } \\
Utilização dos serviços \\
de saúde
\end{tabular}} & \multicolumn{2}{|c|}{ Subcategorias } & \multirow{2}{*}{$\begin{array}{l}\mathbf{n} \\
17\end{array}$} & \multirow{2}{*}{$\begin{array}{r}(\%) \\
(51,5)\end{array}$} \\
\hline & Tipo de serviço & Hospitais e SAP & & \\
\hline & & Centros de Saúde & 14 & $(42,4)$ \\
\hline & & Farmácias & 2 & $(6,1)$ \\
\hline & Motivos de procura & Urgência/acidentes & 6 & $(18,2)$ \\
\hline & & Doenças físicas e psicológicas & 11 & $(33,3)$ \\
\hline & & Vigilância de Saúde & 7 & $(21,2)$ \\
\hline & & Vacinação & 7 & $(21,2)$ \\
\hline & Profissionais a que & Médicos & 33 & $(100)$ \\
\hline & recorrem & Enfermeiros & 6 & $(18,2)$ \\
\hline & & Farmacêuticos & 6 & $(18,2)$ \\
\hline & & Psicólogos & 4 & $(12,1)$ \\
\hline \multirow{5}{*}{$\begin{array}{l}\text { Apreciação negativa } \\
\text { do funcionamento } \\
\text { e condições de } \\
\text { atendimento dos serviços } \\
\text { de saúde }\end{array}$} & Longos períodos de & & 17 & $(51,5)$ \\
\hline & Más condições física & entais/risco de contágio & 7 & $(21,2)$ \\
\hline & Tratam os jovens col & ças/Desvalorizam as suas ideias & 5 & $(15,1)$ \\
\hline & Não respondem às $\mathrm{n}$ & des & 4 & $(12,1)$ \\
\hline & Falta de profissionai & & 4 & $(12,1)$ \\
\hline \multirow{5}{*}{$\begin{array}{l}\text { Preferências em relação } \\
\text { às estruturas dos serviços } \\
\text { de saúde }\end{array}$} & Mais profissionais & & 11 & $(33,3)$ \\
\hline & Atendimento mais $r$ & netódico e horário mais alargado & 10 & $(30,3)$ \\
\hline & Ambiente acolhedor & ável & 7 & $(21,2)$ \\
\hline & Mais recursos mater & & 5 & $(15,2)$ \\
\hline & Adequar os espaços & le população & 3 & $(9,1)$ \\
\hline
\end{tabular}


têm uma doença respiram para cima dos outros... (A5); há grande probabilidade de apanhar doenças (A7). Os horários desadequados e a falta de profissionais também foram aspetos de relevo, nos centros de saúde não respondem às nossas necessidades (A30).

Destaca-se o sentimento de desvalorização das suas ideias ninguém nos ouve (A32), bem como das suas sugestões têm uma caixa de sugestões, mas não mudam nada (A15) associado ao facto de se sentirem tratados como crianças consideram-nos como crianças... não temos idade... (A21).

Ao serem estimulados a falar sobre as suas preferências referiram praticamente o oposto do que identificaram como aspetos negativos no funcionamento dos serviços, destacando-se a necessidade de mais profissionais para satisfazerem uma das condições preferidas como um atendimento mais rápido num horário mais alargado, no sentido de diminuir o tempo de espera e prolongar o horário disponível.

No segundo tema, os adolescentes e os profissionais de saúde, (Tabela 2), são relatadas as experiências concretas de relação com vários grupos profissionais assinalando os aspetos positivos $\mathrm{e}$ negativos referentes a cada um dos grupos que destacaram ${ }^{20}$. Ao abordarem as experiências de atendimento com profissionais de saúde os adolescentes reportam-se sobretudo aos médicos e aos enfermeiros, mas alguns referem também o farmacêutico, como um recurso eficaz e esclarecedor.

Relativamente aos médicos identificaram um conjunto de experiências que remetem quase

Tabela 2. Tema II - Os adolescentes e os profissionais de saúde. Lisboa; 2016.

\begin{tabular}{|c|c|c|c|}
\hline Categorias & Subcategorias & $\mathbf{n}$ & $(\%)$ \\
\hline \multirow{12}{*}{$\begin{array}{l}\text { Experiências de atendimento } \\
\text { com médicos }\end{array}$} & Não sabem lidar com as pessoas & 11 & $(33,3)$ \\
\hline & São negligentes & 9 & $(27,3)$ \\
\hline & Não sabem comunicar & 7 & $(21,2)$ \\
\hline & Preocupam-se e ajudam ${ }^{\star}$ & 5 & $(15,2)$ \\
\hline & Não cumprem regras & 4 & $(12,1)$ \\
\hline & Desvalorizam as queixas & 3 & $(9,1)$ \\
\hline & São estranhos/desconhecidos & 3 & $(9,1)$ \\
\hline & Prescrevem com arbitrariedade & 2 & $(6,1)$ \\
\hline & São disponíveis ${ }^{*}$ & 2 & $(6,1)$ \\
\hline & Informam e esclarecem ${ }^{\star}$ & 2 & $(6,1)$ \\
\hline & São simpáticos ${ }^{\star}$ & 2 & $(6,1)$ \\
\hline & Respeitam a confidencialidade ${ }^{\star}$ & 2 & $(6,1)$ \\
\hline \multirow{4}{*}{$\begin{array}{l}\text { Experiências de atendimento } \\
\text { com enfermeiros }\end{array}$} & Estabelecem boa relação* & 8 & $(24,2)$ \\
\hline & Não sabem lidar com as pessoas & 5 & $(15,2)$ \\
\hline & São compreensivos ${ }^{\star}$ & 2 & $(6,1)$ \\
\hline & Sabem pouco & 2 & $(6,1)$ \\
\hline \multirow{2}{*}{$\begin{array}{l}\text { Experiências de atendimento } \\
\text { com os farmacêuticos }\end{array}$} & São eficazes ${ }^{*}$ & 6 & $(18,2)$ \\
\hline & Informam e esclarecem ${ }^{*}$ & 3 & $(9,1)$ \\
\hline \multirow{13}{*}{$\begin{array}{l}\text { Caracterização do bom } \\
\text { profissional de saúde }\end{array}$} & Ter conhecimentos, ser competente & 17 & $(51,5)$ \\
\hline & Saber relacionar-se (Simpático, atencioso...) & 13 & $(39,4)$ \\
\hline & Ser disponível & 12 & $(36.4)$ \\
\hline & Não tratar os jovens como crianças & 8 & $(24,2)$ \\
\hline & Ter experiência & 7 & $(21,2)$ \\
\hline & Saber informar e explicar & 7 & $(21,2)$ \\
\hline & Gostar de fazer o seu trabalho & 6 & $(18,2)$ \\
\hline & Ser mais velho & 5 & $(15,2)$ \\
\hline & Ser compreensivo & 4 & $(12,1)$ \\
\hline & Ser do sexo feminino & 4 & $(12,1)$ \\
\hline & Transmitir confiança & 4 & $(12,1)$ \\
\hline & Saber respeitar & 3 & $(9,1)$ \\
\hline & Ser pontual & 3 & $(9,1)$ \\
\hline
\end{tabular}


todas para a relação interpessoal e são predominantemente negativas, como não se preocupam com os doentes (A26); são antipáticos, não deram importância às minhas queixas (A33); só receitam medicamentos e não explicam nada (A32); ... às vezes dizem uns nomes que eu não conheço (A5); chegam atrasados (A32). Em contrapartida com alguns aspetos positivos preocuparam-se com as minhas dores (A17) está sempre disponível para discutir a doença comigo (A17).

Para os enfermeiros, a apreciação parece ser mais favorável, destacando-se a capacidade de estabelecer uma boa relação, falam com as pessoas... são mais interativos (A15); existe uma certa confiança entre nós (A16).

Os adolescentes não referiram experiências com psicólogos, todavia reconheceram a sua importância no tratamento de doenças ou problemas psicológicos.

Quando se lhes pediu para caracterizarem $o$ bom profissional de saúde surgiram, a par da importância da relação relacionar-se bem com os jovens (A23); ser atencioso...ter paciência (A3) mostrarse disponível para ajudar (A5), outras dimensões como a competência, os conhecimentos é preciso estar atualizado... ter formação (A9), o saber da experiência saber o que faz... estar treinado (A5), o saber explicar, esclarecer explica bem a doença (A22); falar na nossa linguagem (A7), e compreender os jovens, sem os tratar como crianças $^{20}$.

\section{Fase 2: Grupos Nominais}

Quanto à Fase 2, nos quatro grupos nominais realizados foi produzido, pelos adolescentes no contexto das entrevistas, um conjunto de carac- terísticas e atitudes preferidas nos profissionais de saúde, num total de 116 registos (53 nos dois grupos dos mais novos e 63 nos grupos dos adolescentes mais velhos).

Verificamos, como se pode observar na Tabela 3 , que foram priorizadas 13 características pelos adolescentes mais novos e 11 pelos mais velhos, havendo seis caraterísticas comuns a todos, embora com um posicionamento diferente na ordenação final: ser competente/ter profissionalismo, esclarecer/explicar, ser simpático, ser compreensivo, ser atencioso, ser bem-disposto/divertido, considerando-se globalmente 18 características e/ou atitudes diferentes escolhidas e priorizadas pelos adolescentes.

As caraterísticas que os adolescentes valorizaram incluem a competência e a experiência profissional, a necessidade de informação e esclarecimento de dúvidas (evidentes nos diferentes grupos etários), as questões relacionadas com a confidencialidade e a confiança (no grupo dos mais velhos), passando sempre por uma atitude amigável, compreensiva e facilitadora da relação.

\section{Discussão}

O facto de os adolescentes terem participado ativamente, através das entrevistas de grupo, dando voz às suas ideias permitiu a concretização dos objetivos, e a obtenção de informação relevante sobre as suas preferências relativamente às condições dos serviços e, sobretudo, às atitudes dos profissionais, remetendo para as suas experiências concretas de cuidados de saúde sobretudo com médicos e enfermeiros.

Tabela 3. Características/Atitudes mais importantes nos profissionais de saúde. Lisboa; 2016.

\begin{tabular}{lcclcc}
\hline \multicolumn{1}{c}{ Grupo + Novos } & N=17 & Cotação & \multicolumn{1}{c}{ Grupo + Velhos } & N=14 & Cotação \\
\hline Ser Competente & 11 & 72 & Ter Profissionalismo & 11 & 54 \\
Esclarecer/linguagem clara & 9 & 45 & Explicar de forma clara & 9 & 42 \\
Ser Compreensivo & 9 & 37 & Ser Responsável & 9 & 37 \\
Ser Simpático & 8 & 36 & Ser Disponível & 7 & 28 \\
Ser Prestável & 6 & 28 & Confidencialidade/ Segurança & 7 & 29 \\
Ser Atencioso & 6 & 28 & Ser Simpático & 7 & 25 \\
Ser Educado & 6 & 26 & Ser Compreensivo & 5 & 18 \\
Boa interação/ relação & 6 & 27 & Bom Humor/ Boa Disposição & 4 & 16 \\
Ser divertido & 6 & 18 & Ter vontade de ajudar/ Empenho & 3 & 14 \\
Mostrar interesse & 4 & 18 & Dar voto de confiança & 4 & 15 \\
Preocupar-se & 4 & 18 & Ser atencioso & 2 & 7 \\
Tratar bem /sem arrogância & 3 & 15 & & & \\
Ter postura profissional & 2 & 8 & & &
\end{tabular}


No diálogo com os adolescentes, foi notório o predomínio das referências aos serviços hospitalares e à condição de doença comparativamente à discussão e aspetos relacionados com a saúde e o recurso aos serviços de saúde para vigilância ou promoção da saúde, em concordância com resultados de outros estudos ${ }^{25}$. Quando os aspetos mais preventivos foram abordados, foi principalmente em referência à vacinação, associada à vigilância de saúde. Estes resultados remetem para uma visão predominantemente biomédica, sendo o médico o profissional de referência. Tal pode acontecer pelo facto de tanto os serviços como os profissionais estarem mais direcionados para o tratamento da doença do que para uma visão de promoção da saúde com uma abordagem mais integrativa e holística ${ }^{4}$.

Na globalidade os dados são concordantes com a literatura internacional ${ }^{11-15}$. Os adolescentes expressaram claramente as suas opiniões e expetativas, e manifestaram as suas preferências, com base nas suas experiências com os cuidados de saúde. Houve uma primazia na abordagem das características dos profissionais em relação às condições dos serviços, o que parece traduzir uma maior valorização dos recursos huma$\operatorname{nos}^{13,14,20}$. Contudo, algumas condições dos serviços foram manifestamente valorizadas como negativas, enquanto outras, quase em oposição, se incluíram nas preferências e parecem ser consideradas como condições necessárias à procura dos serviços de saúde pelos adolescentes.

O risco de contágio nos serviços de saúde, nomeadamente nas urgências, foi intensamente valorizado, pressupondo a necessidade de controle da infeção, possivelmente relacionados com as especificidades do desenvolvimento nesta fase, uma vez que os adolescentes estão ainda centrados na causalidade fisiológica da doença por contágio ou contaminação ${ }^{26}$, associadas às condições de uma frequente sobrelotação e escassa higiene dos serviços ${ }^{20}$, frequentemente discutidas nos media.

Surgiram também como importantes as questões da pouca adequação dos serviços às necessidades dos jovens, bem como as questões da acessibilidade $^{25}$, não só pela dificuldade de marcação de consultas, mas também pelo excessivo tempo de espera, frequentemente referido na literatura, e que pode reforçar outros obstáculos à procura dos serviços de saúde pelos adolescentes. Também expressaram um sentimento de desvalorização das suas opiniões e sugestões, resultando no ser tratado como uma criança e uma falta de reconhecimento de um estatuto autónomo dos jovens, o que vem contrariar as tarefas desenvolvimentistas da adolescência ${ }^{26}$.

Os relatos das experiências com os profissionais focaram-se em primeira instância na interação que estes estabelecem com os adolescentes, partindo predominantemente de atitudes negativas para concluírem qual o perfil do bom profissional. O conjunto de resultados aponta, por um lado, para a valorização das atitudes relacionais, de proximidade, simpatia, disponibilidade e ajuda ${ }^{20}$, mas por outro, destaca-se a importância atribuída ao conhecimento, ao tratamento correto e evitamento do erro, à informação associada ao esclarecimento de dúvidas e à clareza da linguagem utilizada na explicação.

Em suma, as competências técnicas surgem reforçadas, fundamentalmente pelos resultados obtidos na segunda fase deste estudo, embora não dissociadas das competências relacionais já emergentes na fase anterior. Estes resultados são semelhantes aos dos estudos pioneiros de Ginsburg e colegas ${ }^{13,14}$. Os adolescentes pretendem profissionais que sejam competentes para tratar as doenças, que os informem esclarecendo as suas dúvidas e que saibam relacionar-se com eles tratando-os como jovens e que, de preferência, gostem de trabalhar com adolescentes.

As preferências expressas foram, em parte, concordantes com a literatura internacional ${ }^{11-15}$, embora com algumas especificidades que se prendem com a realidade dos serviços de saúde do nosso país ${ }^{20}$. As sugestões que emergem dos dados para mudanças futuras assentam em alguns dos critérios de qualidade definidos para os serviços de saúde amigos dos adolescentes $16,17,27$, como o serem mais acessiveis com horários, locais e funcionamento, que permitam o fácil acesso, apropriados ao disponibilizarem serviços adequados às suas necessidades, e aceitáveis quando oferecem cuidados que respondem às expetativas dos adolescentes motivando a procura ${ }^{16,17,20}$. Estes requisitos têm subjacente a filosofia dos cuidados centrados na pessoa do adolescente, uma medida da qualidade de cuidados em saúde ${ }^{18,19}$.

\section{Conclusões}

As entrevistas de grupo permitiram ouvir os adolescentes, dando-lhes a oportunidade de informar, quer os profissionais de saúde quer as entidades políticas, das suas preferências relativamente à necessidade de mudanças.

Conhecer o que pensam, sentem e preferem os adolescentes sobre os cuidados de saúde, per- 
mite compreender melhor as suas perspetivas e saber como adequar os cuidados aos seus desejos, necessidades e preferências, no sentido de estimular o seu interesse e motivação pela procura dos mesmos, e não apenas em condição de doença. Estas mudanças, segundo este grupo de adolescentes, exigem mais recursos humanos, melhores condições de acesso e de atendimento num ambiente físico acolhedor e amigável, facilitador de uma relação próxima e adequada com os profissionais de saúde. Também se evidenciou a necessidade de algumas mudanças nas atitudes e comportamentos dos profissionais, como revelar profissionalismo, ser conhecedor e experiente para esclarecer as dúvidas dos adolescentes e tratar as suas doenças, uma atitude de disponibilidade, simpatia e de compreensão, sem tratar os adolescentes como crianças e mantendo o respeito pela confidencialidade. Estes requisitos transmitem a necessidade do cumprimento dos critérios definidos pela World Health Organization para os "Adolescent Friendly Health Services"16,17.

Salvaguardando as limitações do presente estudo, pela dimensão e região geográfica da amostra, o conjunto dos dados sugere a necessidade de mudanças nas práticas organizacionais e, particularmente, nas atitudes dos profissionais, e vem reforçar a necessidade da implementação do preconizado nas políticas de saúde ${ }^{2,9,10}$ que se pretendem mais ajustadas às suas necessidades e preferências e, por isso, mais centradas no adolescente ${ }^{19}$. Serviços de saúde de elevada qualidade, dirigidos para as necessidades específicas dos adolescentes, podem contribuir para um maior envolvimento dos jovens na sua própria saúde e aumentar a probabilidade de novos atendimentos ${ }^{9}$, facilitando uma transição mais saudável para a idade adulta ${ }^{25}$.

O presente estudo veio também reforçar a adequação da metodologia utilizada na recolha de dados com adolescentes, como alguns autores têm defendido em estudos exploratórios na área da saúde ${ }^{20,23}$. Dar voz aos jovens em grupo estimulou o seu pensamento e a sua participação ativa sobre questões relativas à sua saúde. De referir, o benefício de o estudo ter incluído uma primeira fase de expressão mais livre e uma segunda fase mais regulada e estruturada, permitindo não só a emergência de novos dados como o reforço de outros já recolhidos antes, bem como ter confirmado a importância da continuidade da investigação nesta área.

\section{Colaboradores}

MG Vinagre e L Barros participaram na conceção e desenho metodológico do estudo e na revisão final do artigo. MG Vinagre efetuou a revisão de literatura, a recolha, tratamento e análise dos dados e assumiu a redação do artigo e L Barros participou na análise e interpretação dos dados e fez a revisão crítica do artigo.

\section{Agradecimentos}

Agradecemos a todos os adolescentes que participaram no estudo e aos pais que o permitiram, bem como aos Conselhos Executivos das escolas e a todos os professores que aceitaram colaborar.

Trabalho financiado pela Fundação para a Ciência e a Tecnologia (FCT). 


\section{Referências}

1. Dick B, Ferguson J. Health for the World's adolescents: a second chance in the second decade. J Adolesc Health 2015; 56(1):3-6.

2. Direção-Geral da Saúde (DGS). Programa Nacional de Saúde Infantil e Juvenil. Lisboa: DGS; 2013.

3. Patton GC, Sawyer SM, Ross DA, Viner RM, Santelli JS. From advocacy to action in global adolescent health. $J$ Adolesc Health 2016; 59(4):375-377.

4. Aquino J, Miranda P, Luz A, Moleiro P. O perfil biopsicossocial do adolescente em consulta hospitalar-experiência de 8 anos de uma unidade de medicina do adolescente. Rev Psicol Cr Adolesc 2015; 6(2):31-46.

5. Weiss AL, D'Angelo LJ, Rucker AC. Adolescent use of the emergency department instead of the primary care provider: who, why, and how urgent? J Adolesc Health 2014; 54(4):416-420.

6. Administração Regional de Lisboa e Vale do Tejo. Análise do movimento assistencial dos cuidados de saúde primários e consultas e urgências hospitalares: 2012/2013. Lisboa: M. Saúde; 2014.

7. Matos, MG. Novos rumos na educação e promoção da saúde a partir de uma reflexão sobre a intervenção com crianças e adolescentes no Aventura Social. Saúde \& Tecnologia 2014; 12:5-7.

8. Hargreaves DS, Graves F, Levay C, Mitchell I, Koch U, Esch T, Denny S, Frich JC, Struijs J, Sheikh A. Comparison of health care experience and access between young and older adults in 11 high-income countries. J Adolesc Health 2015; 57(4):413-420.

9. Yassaee AA, Hargreaves DS, Chester K, Lamb S, Hagell A, Brooks FM. Experience of primary care services among early adolescents in England and association with health outcomes. J Adolesc Health 2017; 60(4):388-394

10. Brasil. Ministério da Saúde (MS). Secretaria de Atenção à Saúde. Departamento de Ações Programáticas e Estratégicas. Proteger e cuidar da saúde de adolescentes na atenção básica. Brasília; 2017. [acessado 2018 Fev 20]. Disponível em: http://editora.saude.gov.br

11. Vingilis, E, Wade T, Seeley J. Predictors of adolescent health care utilization. J Adolesc 2007; 30:773-800.

12. Freake H, Barley V, Kent G. Adolescents' views of helping professionals: A review of literature. J Adolesc. 2007; 30(4):639-653.

13. Ginsburg KR, Forke CM, Cnaan A, Slap GB. Important health provider characteristics: the perspective of urban ninth graders. J Dev Behav Pediatr 2002; 23(4):237243.

14. Ginsburg K R, Menapace A, Slap GB. Factors affecting the decision to seek health care: The voice of adolescents. Pediatrics 1997; 100(6):922-930.

15. Lambert V, Coad J, Hicks P, Glacken M. Young children's perspectives of ideal physical design features for hospital-built environments. J Child Health Care. 2014; 18(1):57-71.
16. World Health Organization (WHO). Making health services adolescent friendly: developing national quality standards for adolescent-friendly health services. Geneva: WHO; 2012.

17. World Health Organization (WHO). Global standards for quality health-care services for adolescents: a guide to implement a standards-driven approach to improve the quality of health care services for adolescents (vol.1):Standards and criteria. Geneva: WHO; 2015.

18. Toomey SL, Elliott MN, Schwebel DC, Tortolero SR, Cuccaro PM, Davies SL, Kampalath V, Schuster MA. Relationship between adolescent report of patient-centered care and of quality of primary care. Acad. Pediatr. 2016; 16:770-776.

19. Hargreaves DS. Learning to listen: delivering patient-centered care for adolescents. J Adolesc Health 2014; 55(4):463-464.

20. Vinagre MG, Barros L. Os olhares de um grupo de adolescentes sobre os profissionais e os serviços de saúde. In: Atas do $6^{\circ}$ Congresso Ibero-Americano em Investigação Qualitativa: Investigação Qualitativa em Saúde; 2017; Salamanca. p. 372-381.

21. Hargreaves, DS, Viner RM. Children's and young people's experience of the National Health Service in England: a review of national surveys 2001-2011. Arch Dis Child 2012; 97:661-666.

22. Morgan DL. The focus group guidebook. Thousand Oaks: Sage Publications; 1998.

23. Macphail A. Nominal group Technique: a useful method for working with young people. Br Educ Res J 2001; 27(2):161-170

24. Bardin L. Análise de conteúdo. Lisboa: Edições 70; 2011.

25. Nunes BP, Flores TR, Duro, SMS, Saes MdO, Tomasi E, Santiago AD, Thumé E, Facchini LA. Utilização dos serviços de saúde por adolescentes: estudo transversal de base populacional, Pelotas-RS 2012. Epidemiol. Serv. Saúde 2015; 24(3):411-420.

26. Barros L. Psicologia Pediátrica: perspetiva desenvolvimentista. $2^{\text {a }}$ ed. Lisboa: Climepsi; 2003.

27. Ambresin A-E, Michaud P-A, Haller DM. Services "amis des jeunes»: promouvoir des soins adaptés aux adolescentes. Rev Méd Suisse 2014; 10(434):1278-1281.

Artigo apresentado em 02/04/2018

Aprovado em 22/10/2018

Versão final apresentada em 19/02/2019 